\title{
Lactoferrin reduces mortality in preweaned calves with diarrhea
}

\author{
G. Habing, ${ }^{* 1}$ K. Harris, ${ }^{*}$ G. M. Schuenemann, ${ }^{*}$ J. M. Piñeiro, ${ }^{*}$ J. Lakritz,† and X. Alcaraz Clavijoł \\ *Department of Veterinary Preventive Medicine, and \\ †Department of Veterinary Clinical Sciences, College of Veterinary Medicine, The Ohio State University, Columbus 43210 \\ ‡Universidad Nacional de Columbia, Bogota, Columbia
}

\begin{abstract}
Calf diarrhea is the most common reason for mortality and antimicrobial therapy in preweaned calves on dairy farms in the United States. Conventional and organic livestock producers require alternative therapies for calf diarrhea to reduce the necessity of conventional antimicrobials. Alternatives administered for mild cases or early in the disease course may be useful to mitigate disease progression and reduce the likelihood of septicemia and negative sequelae. Lactoferrin is a bioactive protein naturally found in colostrum that has been shown to prevent septicemia in high-risk infants. Among organic producers, garlic extract is widely used for the treatment of disease and perceived to be efficacious. The objectives of the study were to determine the effectiveness of lactoferrin and garlic extract to reduce mortality and culling, improve weight gain, and reduce the duration of disease in preweaned calves with the first diagnosis of diarrhea. In total, 628 calves with diarrhea from a single commercial dairy were enrolled in a blinded, randomized field trial. Calves diagnosed with diarrhea (fecal score $\geq 3$ ), were randomized to 3 consecutive days of oral garlic extract, lactoferrin, or water (control). Calves were clinically evaluated for up to $10 \mathrm{~d}$. Body weight was measured at enrollment and $10 \mathrm{~d}$ later. For calves receiving garlic extract, the risk of death or culling was not significantly different than calves in the control group; however, calves that received lactoferrin had approximately half the risk of death or culling in the $120 \mathrm{~d}$ following diagnosis. Additionally, the relative risk of death or culling in the $60 \mathrm{~d}$ following diagnosis was significantly lower for the subset of calves with severe diarrhea at enrollment. Neither garlic nor lactoferrin had a significant effect on disease duration or average weight gain during the $10-d$ period. Lactoferrin significantly reduced mortality and culling when administered to preweaned calves with the first diagnosis of diarrhea; however, additional studies conducted across multiple farms are necessary
\end{abstract}

Received September 7, 2016.

Accepted January 18, 2017.

${ }^{1}$ Corresponding author: habing.4@osu.edu to corroborate the observed reduction in mortality and culling. If the results are confirmed, lactoferrin may become an important tool to improve treatment outcomes and reduce the necessity of antimicrobials.

Key words: calf diarrhea, lactoferrin, calf mortality, antimicrobial alternative, garlic extract

\section{INTRODUCTION}

Epidemiologic linkages between antimicrobial use in livestock and antimicrobial resistance in human pathogens have prompted increasing public and regulatory pressure for reduced livestock antimicrobial use (Dutil, 2010; Saini et al., 2012). However, efforts to restrict antimicrobial use must be balanced against the need to protect livestock health. Alternative therapies are needed to reduce the necessity of conventional antimicrobials important to human health. Additionally, organic livestock producers require alternative therapies to meet stringent USDA requirements that prohibit antimicrobial use within organically certified animals. Avoidance of antimicrobial use is particularly challenging within calf-production systems, where large populations of highly susceptible neonates facilitate rapid transmission of disease. Calf diarrhea is the most common reason for mortality and usage of antimicrobial therapy in preweaned calves on dairy farms in the United States (USDA, 2010; Lombard et al., 2015), and occurs frequently on both conventional and organic farms (Habing et al., 2016). The associated small intestinal overgrowth of Escherichia coli and the reasonable likelihood of septicemia in some cases warrants parenteral antimicrobial therapy (Fecteau et al., 1997; Lofstedt et al., 1999; Constable, 2004). However, alternatives to antimicrobial drugs with proven efficacy and administered for mild cases or early in the disease course may be useful to mitigate disease progression, reduce the likelihood of septicemia, and reduce the necessity of antimicrobial use.

Lactoferrin is a bioactive protein naturally found in colostrum that has antimicrobial, antiviral, and immunomodulatory effects (Elass-Rochard et al., 1998; Ellison and Giehl, 1991; Berlutti et al., 2011). It has been extensively investigated as a prophylactic to prevent 
sepsis in high-risk infants (Weinberg, 2001; Zavaleta et al., 2007; Turin et al., 2014). As approximately $30 \%$ of calves with severe diarrhea have septicemia (Fecteau et al., 1997; Lofstedt et al., 1999), lactoferrin may also be useful to prevent septicemia when administered to high-risk calves before or early in the course of disease; however, prior studies have evaluated lactoferrin as a supplement, and have either found a positive effect on weight gain or disease prevention (van Leeuwen et al., 2000; Joslin et al., 2002; Robblee et al., 2003; Prenner et al., 2007) or no significant difference (Cowles et al., 2006; English et al., 2007). An experimental challenge trial demonstrated that lactoferrin and lactoperoxidase effectively mitigated the negative health outcomes in calves inoculated with pathogenic E. coli (Still et al., 1990). No studies have examined the effect of lactoferrin when administered as a treatment for naturally occurring diarrhea. A randomized clinical trial with naturally occurring cases is necessary to test the effects of lactoferrin as when administered a treatment for calf diarrhea.

Organic producers often rely on natural alternatives to conventional antimicrobials for the treatment of disease, including garlic, aloe, or other herbal therapies (Habing et al., 2016). For instance, approximately 68\% of organic producers reported that they used garlic for the treatment of calves with diarrhea, and $>75 \%$ perceived garlic treatments to be efficacious (Habing et al., 2016). Allicin (garlic) is known to have of in vitro activity against a wide range of gram-negative bacteria, and prior field trials have demonstrated improved growth in calves with dietary garlic extract supplementation (Ghosh et al., 2011, 2010), Therefore, the plausible effectiveness and the widespread use of garlic extract by organic producers warrants a randomized clinical trial to facilitate the creation of evidence-based treatment protocols.

Randomized clinical trials testing novel therapeutics can be used to construct evidence-based treatment protocols for use on the farm. The overall objective of our research was to use a randomized clinical trial to determine the effectiveness of lactoferrin and garlic extract for the treatment of calf diarrhea. We hypothesized that lactoferrin or garlic extract would reduce mortality and culling, improve weight gain, and reduce the duration of disease in preweaned calves with the first diagnosis of diarrhea.

\section{MATERIALS AND METHODS}

\section{Setting}

The trial was conducted on a single large organic dairy calf ranch in the western United States. All ac- tivities were approved by the Ohio State University (OSU) Institutional Animal Care and Use Committee (2013A00000054). Holstein calves used in this study were born within a single calving facility on a certified organic dairy operation. Fresh colostrum was harvested at the maternity within 1 to $2 \mathrm{~h}$ after calving and all calves received $4 \mathrm{~L}$ of optimum quality colostrum (colostrometer; > $50 \mathrm{IgG} \mathrm{mg} / \mathrm{dL}$ ) within $4 \mathrm{~h}$ after birth either by suckling or via oro-esophageal feeder. Colostrum not immediately fed to calves was refrigerated at $4^{\circ} \mathrm{C}$ in individual bottles (not pooled) and fed to calves with 2 $\mathrm{d}$ of collection. All calves were transported to the calf ranch within $24 \mathrm{~h}$ of birth and housed in individual outdoor hutches. For the first 2 feedings, calves were bottle-fed colostrum, and thereafter were bucket-fed twice daily a total of $8 \mathrm{~L} / \mathrm{d}$ of whole pasteurized milk. A commercial starter grain mix was offered to calves beginning at the third day of life. The targeted weaning age was $56 \mathrm{~d}$, and groups of calves were weaned weekly. At weaning, the milk feedings were reduced to once daily for 1 wk. Weaned calves were moved to group housing and received commercial starter grain for $1 \mathrm{wk}$, and were then fed a TMR.

\section{Inclusion/Exclusion Criteria}

All calves born on the dairy operation and transported to the calf ranch between May 15 and September 16,2015 , were visually assessed in the morning of each day by OSU personnel for the first 3 wk of life. Fecal scores were recorded as a 1 (formed), 2 (semi-formed, pasty), 3 (loose but stays on top of the bedding), or 4 (watery diarrhea that sifts through the bedding). Dehydration scores were recorded as a 1 (no signs of dehydration), 2 (mild depression, skin tent in the neck region of $2-6 \mathrm{~s}$, recessed eyes), 3 (skin tent $>6 \mathrm{~s}$, very recessed eyes, and the calf unwilling or reluctant to get up), or 4 (calf will not stand, skin does not flatten when tented; McGuirk, 2008). Calves were enrolled after they were diagnosed by OSU personnel for the first time, with diarrhea (fecal score of 3 or 4). Calves with severe dehydration (dehydration score $=2$ ), with concurrent significant disease (e.g., pneumonia, umbilical infections), or calves that required immediate antimicrobial therapy were excluded. Calves excluded because of pneumonia were those that had an elevated rectal temperature $(>39.4 \mathrm{C})$ with either repeated spontaneous coughs or excessive mucus nasal discharge. Calves excluded due to umbilical disease had an enlarged and painful umbilicus with or without an elevated rectal temperature.

Treatments for calves with diarrhea (e.g., fluids, antiinflammatories) were administered by farm personnel according to the farm protocols, but study treatments 
(lactoferrin, garlic extract, and control) were administered by OSU study personnel. The farm protocol for routine diarrhea cases consisted of administration of an oral fluid electrolyte solution of $1 \mathrm{~L}$ twice per day of Re-Sorb (Zoetis Animal Health, Florham Park, NJ), with continuation of the daily milk feeding program for all calves. Additionally, according to farm protocol, calves with a rectal temperature $>39.4^{\circ} \mathrm{C}$ or with apparent blood in the feces received $2.2 \mathrm{mg} / \mathrm{kg}$ i.v. of flunixin meglumine once per day for $3 \mathrm{~d}$. Calves with an estimated percent dehydration $>6 \%$ received 1 to $2 \mathrm{~L}$ of lactated ringers solution i.v. with or without additional sodium bicarbonate.

The study was designed to provide sufficient statistical power to demonstrate a difference in mortality and culling between groups. Assuming a 13\% mortality and culling rate and a $62 \%$ reduction as a result of treatment, 200 cases/group will provide sufficient power $(0.80)$ to detect a significant $(\alpha=0.05)$ difference.

\section{Treatments}

Lactoferrin was obtained as a freeze-dried protein purified from bovine milk. The product analysis certificate listed the product as $98.5 \%$ protein, a protein composition of $91.8 \%$ lactoferrin, and an iron saturation of $11 \%$. Lactoferrin treatments were prepared by mixing $30 \mathrm{~mL}$ of distilled water with $3 \mathrm{~g}$ of lactoferrin. Frozen garlic extract was prepared according to previously described methods (Ghosh et al., 2011). Briefly, fresh certified organic garlic was added to distilled water in a 1:2 weight-to-volume ratio and blended in a high-speed blender for $2 \mathrm{~min}$. The solids were separated using a filter (muslin cloth), and 30-mL aliquots of the resultant liquid were immediately frozen in sterile bags. Calves meeting the criteria for enrollment received either $30 \mathrm{~mL}$ of water containing $3 \mathrm{~g}$ of lactoferrin, $30 \mathrm{~mL}$ of extract equivalent to $10 \mathrm{~g}$ of whole garlic, or $30 \mathrm{~mL}$ of water (control) administered orally once daily for 3 consecutive days.

\section{Treatment Allocation and Blinding}

To balance treatment allocation over time and withinfarm locations, calves were sequentially randomized to 1 of the 3 treatment groups in blocks of 3 , and the within-block order was randomized to prevent anticipation of the treatment allocation. This randomization sheet was created using Microsoft Excel (Microsoft Corp., Redmond, WA). To limit the awareness of investigators to treatment assignments, new cases were enrolled and health assessments were performed by OSU personnel before treatment allocation and administration. After completion of assessments, the necessary number of treatments was prepared and opaque tape labeled with the calf identification was used to render the syringes visually indistinguishable. Although the garlic could be smelled, investigators administered a median number of 15 visually indistinguishable syringes within a short time frame, and thus it was difficult to associate the constant smell of garlic to the treatment assignment of a specific calf. Therefore, due to the arrangement of the work order, the large number of treatments administered, and the use of visually indistinguishable oral syringes, OSU study personnel were not aware of the treatment assignments during the subjective health assessments and treatment administration.

\section{Study Outcomes}

The 3 primary study outcomes were 60 -d mortality and culling rate, weight gain in the $10 \mathrm{~d}$ following enrollment, and duration of disease. Calves were weighed at enrollment and $10 \mathrm{~d}$ following enrollment using a digital scale. Scale weights were not available for the first $11 \mathrm{~d}$ of the study, and these calves were not included in this portion of the analysis. To measure disease duration, daily health assessments were performed for a maximum of $10 \mathrm{~d}$ following enrollment, or until calves had 2 consecutive days with normal clinical parameters. At each assessment, OSU study personnel completed a physical exam and recorded the fecal score (4-point scale), dehydration score (4-point scale), and rectal temperature and noted any other clinical abnormalities. Farm records were retrieved to identify calves that died or were culled in the 10,60, or $120 \mathrm{~d}$ following enrollment. The cause of death was not determined by OSU personnel; however, farm personnel categorized causes of death as either diarrhea- or respiratory-associated. Calves were culled if farm personnel deemed that the severity of disease necessitated antimicrobial therapy. Decisions on treatment and culling were made as per existing farm protocols, without the consultation of OSU personnel on the farm.

\section{Statistical Analysis}

Means, proportions, and standard errors were calculated for baseline characteristics of calf diarrhea cases. Enrollment of calves into the lactoferrin treatment group was delayed for 2 wk awaiting the organic approval process, and enrollment of calves into the control and lactoferrin groups was extended 2 wk beyond the enrollment of calves into the garlic treatment group. To avoid bias, statistical comparisons between lactoferrin and control and between garlic and control groups 
were made separately and restricted to time periods of contemporaneous enrollment of treatment and control group. A linear mixed model (PROC MIXED, SAS v. 9.4, SAS Institute Inc., Cary, NC) was used to test for baseline treatment group differences in weight at enrollment, final weight, and ADG. A chi-squared test was used to test for differences in the proportion of calves with a fecal score of 4 or a dehydration score of 2. Age at enrollment was compared between groups using a nonparametric ANOVA (Wilcoxon scores with Kruskal-Wallis test). Disease duration was defined as the number of days between enrollment and the first day of 2 consecutive days with normal health assessment parameters. Calves were defined as clinically normal when they had a fecal score of 1 or 2 , a dehydration score of 1 , and rectal temperature $<39.4^{\circ} \mathrm{C}$. Calves were right censored within the survival analysis if there were 2 consecutive days where the health assessments were not performed. In other words, censored calves did not experience the event of interest (clinical resolution), but were considered to have been abnormal up to the time of censoring. A cox proportional hazards model (PROC PHREG, SAS v. 9.4) was used to identify differences in the number of days to clinical resolution. The assumptions for proportional hazards were assessed by visualizing the survival function plot for each treatment group. Calves that were enrolled but subsequently developed severe disease requiring antimicrobial therapy were culled from the production system; therefore, the mortality outcome is unknown. Mortality and culling are descriptively compared across treatment groups; however, as both represent a failure of the calf to survive on the farm, the sum of deaths and culls is used for the statistical analysis. A Poisson regression model (PROC GENMOD, SAS v. 9.4) with robust standard errors (Zou, 2004) was used to test differences in the incidence risk of death or culling before 10,60, and 120 $\mathrm{d}$ between treatment groups. Relative risks of death or culling and the associated $95 \%$ confidence intervals for the lactoferrin and garlic treatment groups were derived from the model using the estimate statement. To assess for potential confounding by case characteristics at enrollment, variables that reflected signalment or severity at enrollment (age, rectal temperature, and fecal score) were offered to the model and retained if the percent difference in the treatment effect (relative risk) with and without inclusion of the covariate exceeded $20 \%$. Furthermore, the interaction between treatment and the same baseline case characteristics was assessed to identify effect modification and describe treatment effects across case characteristics at enrollment (e.g., fever or no fever). Statistical significance of the model parameters were tested using the Wald Chi-Square test.

\section{RESULTS}

\section{Enrollment}

Between May 15 and September 16, 2015, 1,534 calves were brought to the calf ranch, and 628 calves (41\%) met the criteria for enrollment within the first 3 wk on the farm. A median of 5 calves (range 1-11) were enrolled per day, but more than 3 calves were enrolled on $89 \%(108 / 122)$ of the enrollment days. The median age at enrollment was $11 \mathrm{~d}$, and ranged from 1 to 32 d of age (Figure 1). Initially, 630 calves were enrolled; however, 2 calves were enrolled more than once (multiple episodes of diarrhea), and only the first enrollment was included in the analysis. Four calves over the 21-d age inclusion criteria were inadvertently enrolled, but remained in the analysis to stay consistent with an intent-to-treat analysis approach. In total, 199, 205, and 224 calves with diarrhea were enrolled into the lactoferrin, garlic extract, and control groups, respectively. To account for the delay in lactoferrin enrollments, statistical comparisons between lactoferrin and control were restricted to 199 and 198 calves in each respective group (time period of contemporaneous enrollment). Statistical comparisons between garlic and control were restricted to 201 and 198 calves, respectively. Baseline characteristics, including age, initial weight, percent with an initial fecal score of 4 , and percent with an

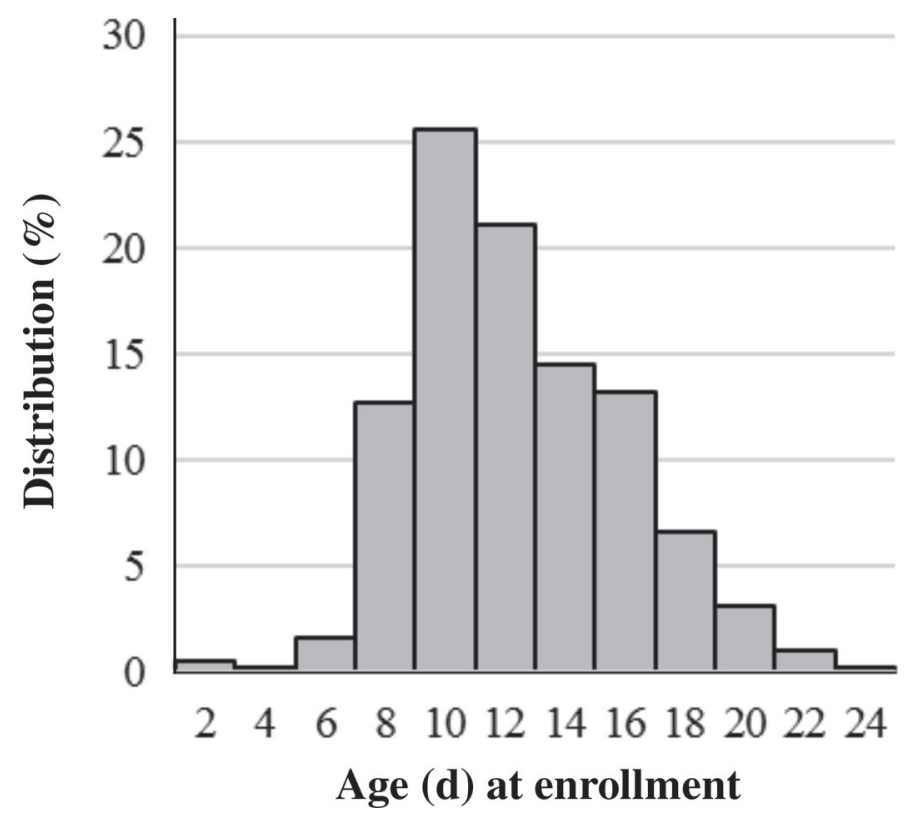

Figure 1. Age at enrollment for preweaned calves with the first diagnosed case of diarrhea in a randomized clinical trial of lactoferrin and garlic extract for the treatment of calf diarrhea. 
Table 1. Number (\%) of calves that were treated with lactoferrin, garlic extract, or a control for the first diagnosis of diarrhea that died or were culled within the subsequent 10,60 , or $120 \mathrm{~d}$

\begin{tabular}{|c|c|c|c|c|c|c|c|c|c|}
\hline \multirow[b]{2}{*}{ Treatment } & \multicolumn{3}{|c|}{$10 \mathrm{~d}$} & \multicolumn{3}{|c|}{$60 \mathrm{~d}$} & \multicolumn{3}{|c|}{$120 \mathrm{~d}$} \\
\hline & Died & Culled & Total & Died & Culled & Total & Died & Culled & Total \\
\hline Garlic $(\mathrm{n}=205)$ & $5(2.4)$ & $2(1.0)$ & $7(3.4)$ & $8(3.9)$ & $13(6.3)$ & $21(10.2)$ & $14(6.8)$ & $18(8.8)$ & $32(15.6)$ \\
\hline Control $(\mathrm{n}=224)$ & $8(3.6)$ & $6(2.7)$ & $14(6.3)$ & $15(6.7)$ & $16(7.1)$ & $31(13.8)$ & $22(9.8)$ & $22(9.8)$ & $44(19.6)$ \\
\hline
\end{tabular}

initial dehydration score of 2 did not significantly differ between treatment groups $(P>0.05)$.

\section{Risk of Death and Culling}

For all calves enrolled, $10.5 \%(66 / 628)$ died or were culled before $60 \mathrm{~d}$ of age, and $14.8 \%(93 / 628)$ died or were culled prior to $120 \mathrm{~d}$ of age (Table 1 ). The model effect estimates for lactoferrin or garlic were not substantially (i.e., >20\%) different when including baseline case characteristics (age, fecal score, dehydration score) into the model, suggesting that case severity was evenly distributed between treatment groups. Therefore, treatment effects unadjusted for baseline characteristics are reported. Calves that received lactoferrin were approximately half as likely (relative to control) to die or be culled within $120 \mathrm{~d}$ following diagnosis $(P<0.05)$ (Figure 2). For calves in lactoferrin treatment group, the unadjusted relative risk of death or culling before 10,60 , and $120 \mathrm{~d}$ following diagnosis was $0.60(95 \%$ CI: $0.22-1.51), 0.58$ (95\% CI: $0.31-1.09)$ and 0.48 (95\% CI: $0.28-0.83$ ), respectively (Figure 2 ). For calves receiving garlic extract for the treatment of diarrhea, the 10-, 60-, and 120-d risk of death or culling was not significantly different than calves in the control group (Table 1). For the subset of calves with a fecal score of 4 (watery diarrhea) at enrollment, $16.5(13 / 79)$ and $3 \%$ $(2 / 63)$ of calves in the control and lactoferrin groups, respectively, died or were culled in the following $60 \mathrm{~d}$ $(P<0.05)$. In the model, the interaction between treatment group and initial fecal score was significant $(P$ $=0.02$ ), suggesting that the treatment effect (relative risk) was significantly different across different fecal scores. For calves with a fecal score of 3 , the relative risk (lactoferrin vs. control) of death or culling within $60 \mathrm{~d}$ of enrollment for calves was 0.95 (95\% CI: 0.42 $2.07)$. For calves with a fecal score of 4 , the relative risk was 0.19 (95\% CI: 0.05-0.82; Figure 3). We found no significant interaction with dehydration score or rectal temperature at enrollment $(P>0.50)$. The effect of lactoferrin on the risk of death or culling was still apparent between 60 and $120 \mathrm{~d}$ following enrollment. The risk of death between 60 and 120 d was $1.6 \%(3 / 185)$ in the lactoferrin group compared with $9 \%(18 / 198)$ in the control group $(P=0.002)$. The median age at death was 38.5, 52, and $14 \mathrm{~d}$ for calves in the control $(\mathrm{n}=22$ deaths $)$, garlic $(\mathrm{n}=14$ deaths $)$ and lactoferrin ( $\mathrm{n}=6$ deaths), respectively. The lower median age at death for lactoferrin calves was due to a low number of deaths $>10 \mathrm{~d}$ postenrollment, rather than a higher risk for death closer to the episode of diarrhea. Of the 42 recorded deaths, 36 (86\%) were recorded by farm personnel as associated with respiratory disease, and 3 (7\%) were reported as associated with gastrointestinal disease.

\section{Weight Gain}

Weight gain in the $10 \mathrm{~d}$ following diagnosis was measured for 188, 170, and 182 calves in the control, garlic, and lactoferrin treatment groups, respectively. Across all treatment groups, the mean (SE) weight at enrollment and $10 \mathrm{~d}$ following enrollment was $40.2( \pm 0.17)$ and $43.0 \mathrm{~kg}( \pm 0.19)$, respectively (Table 2$)$. Initial weight and final weight in the 10-d subsequent diag-

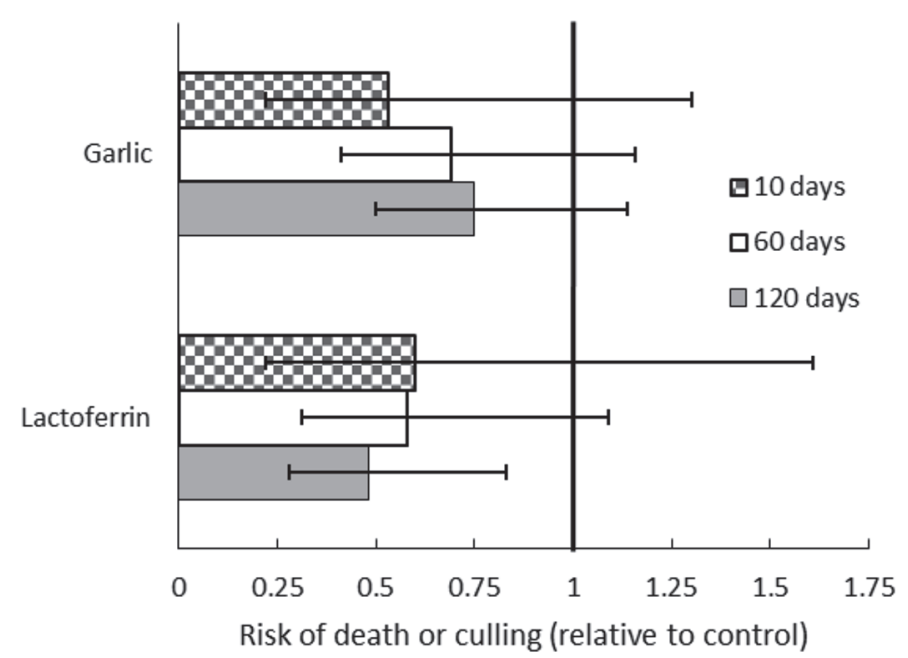

Figure 2. Effect of treatment with lactoferrin and garlic extract on the relative risk $(95 \% \mathrm{CI})$ of death in the 10,60 and $120 \mathrm{~d}$ following the first diagnosis of diarrhea. 


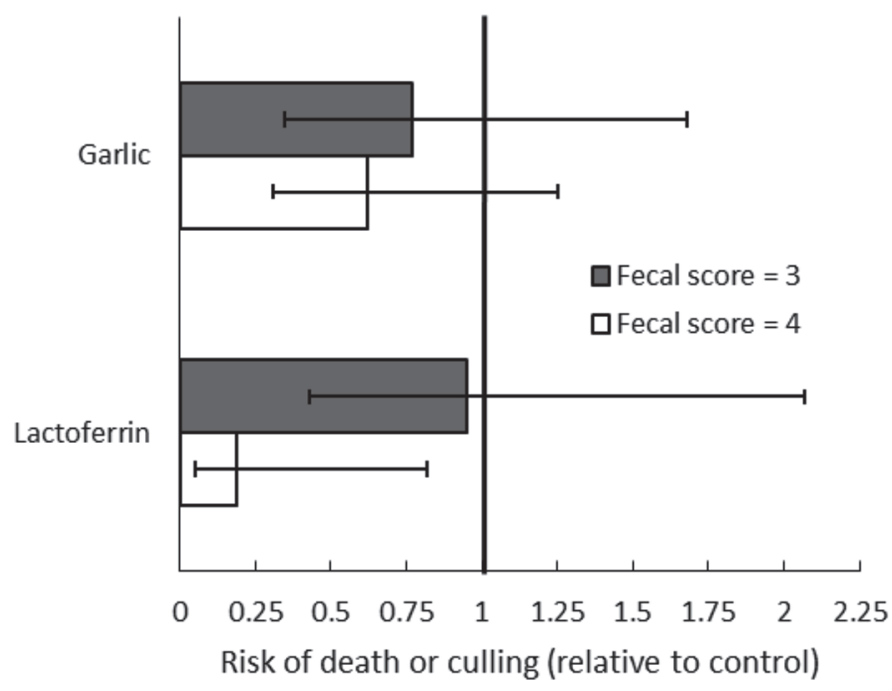

Figure 3. Effect of treatment with lactoferrin and garlic extract on the relative risk $(95 \% \mathrm{CI})$ of death in the $60 \mathrm{~d}$ following the first diagnosis of diarrhea for calves where the initial fecal score was either 3 (diarrhea that does not sift through the bedding) or 4 (watery diarrhea that sifts through the bedding).

nosis were not significantly $(P>0.05)$ different across treatment groups. The mean $\mathrm{ADG}(\mathrm{SE}=0.01)$ were $0.30,0.29$, and $0.29 \mathrm{~kg} / \mathrm{d}$ for calves in the lactoferrin, garlic extract and control groups, respectively, and was not significantly different $(P=0.81)$.

\section{Disease Duration and Progression}

In total, $80.5 \%$ (505/628) calves were defined as clinically normal before the end of the 10-d follow-up period. Of the remaining 123 calves, 44 had complete data but never met the criteria, 28 died or were sold before the end of the 10-d follow-up period, and 51 were censored before completion of the 10-d follow-up period. Plots of the survival function were similar across treatment groups. Based on the Cox proportional hazard model results, disease duration was not significantly associated with treatment group. The 25th, 50th, and 75th percentile for the duration of disease was 1,3 , and 6

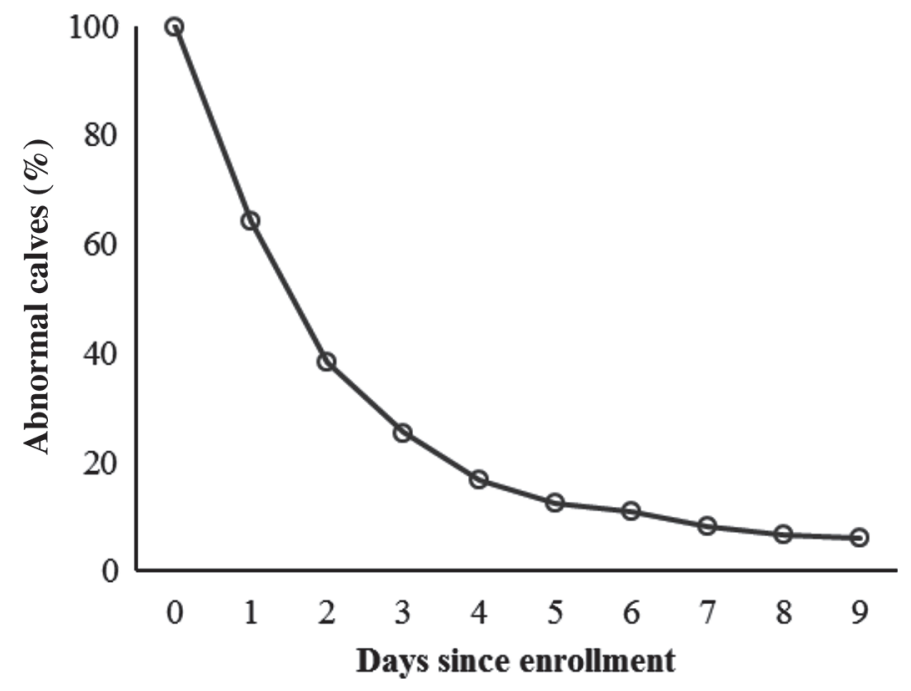

Figure 4. Proportion of calves with abnormal clinical characteristics in the $9 \mathrm{~d}$ following the first diagnosis of diarrhea. Calves were classified as normal on the first day of 2 consecutive days of a normal fecal score, dehydration score, and rectal temperature. We found no significant difference in duration of disease between treatment groups.

d, respectively (Figure 4). Additionally, we found no difference between treatment groups in the progression from milder signs to more severe signs during the 10-d follow-up period. Among calves enrolled without signs of dehydration (dehydration score = 1), $19(41 / 214$ ), $13(26 / 196)$, and $16 \%(32 / 196)$ of calves in the control, garlic, and lactoferrin treatment groups, respectively, had at least 1 subsequent day with a dehydration score of 2 within the 10-d follow-up period. Among calves enrolled into the trial without a fever, 20 (34/172), 19 $(31 / 164)$, and $15 \%(24 / 162)$ calves in the respective groups had at least $1 \mathrm{~d}$ with a recorded fever in the 10-d follow-up period.

\section{DISCUSSION}

Randomized controlled trials are necessary to identify effective alternatives to conventional antimicrobials and substantiate anecdotal reports of efficacy for non-

Table 2. Characteristics of calves at enrollment in a randomized clinical trial on the effect of lactoferrin and garlic on the treatment of calves for diarrhea

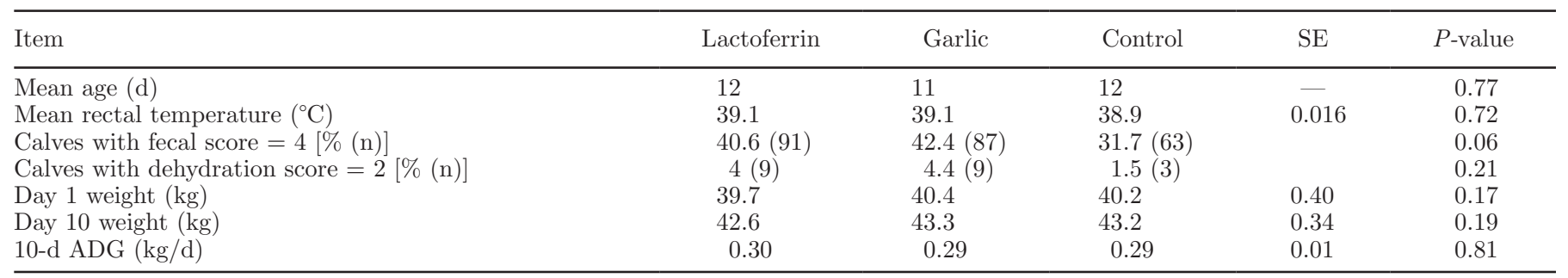


traditional therapies (Habing et al., 2016). Targeting antimicrobial therapy in calves may reduce costs and benefit overall calf health (Berge et al., 2009). Medical literature suggests that lactoferrin reduces the risk of sepsis in high-risk infants, but field studies have not tested the efficacy of lactoferrin for the treatment of diarrhea in neonatal calves. Likewise, garlic extract is widely used and perceived to be efficacious by organic dairy producers (Habing et al., 2016); however, the effectiveness of garlic as a therapy for calf diarrhea has not been tested. Results from the current study suggest that lactoferrin significantly reduces the risk of death or culling when used as a treatment for preweaned calves with the first diagnosis of diarrhea. These results suggest lactoferrin can be included in treatment protocols on organic and conventional farms to reduce reliance on conventional antimicrobials and improve calf health.

A prior experimental trial that demonstrated that the combination of lactoferrin and lactoperoxidase mitigated the negative effects of an experimental challenged with pathogenic strains of E. coli (Still et al., 1990). Lactoferrin may also effectively mitigate the small intestinal bacterial overgrowth of $E$. coli, which occurs regardless of the initiating cause of diarrhea (Moon et al., 1978; Constable, 2004). The current study suggests that that lactoferrin improves long-term health outcomes using naturally occurring cases on a commercial dairy farm. The current study also suggests a larger treatment effect with more severe cases of diarrhea. The estimated relative risk of death or culling was 0.95 and 0.19 for calves with an initial fecal score of 3 and 4 , respectively, suggesting that lactoferrin may be best targeted to calves with more watery diarrhea. Additional strengths of our study include the robust randomization and blinding procedures that reduced the likelihood of bias. Nonetheless, additional studies are necessary to confirm the findings and identify potential mechanisms for the observed reductions in mortality and culling.

Lactoferrin may reduce the incidence of septicemia in diarrheic calves by binding LPS, which may interfere with LPS-induced cellular signaling associated with inflammation (Baveye et al., 2000) and could prevent attachment of E. coli to enteric cells (Flores-Villaseñor et al., 2012). Lactoferrin also exerts antimicrobial activity by binding iron and limiting the availability of iron for bacterial growth (Ellison and Giehl, 1991). Therapies with in vitro activity against E. coli (including both garlic extract and lactoferrin) may mitigate the small intestinal bacterial overgrowth of $E$. coli, which often occurs in diarrheic calves (Isaacson et al., 1978; Moon et al., 1978). Because of lactoferrin is a protein, calves enrolled into this treatment group received an addition- al $9 \mathrm{~g}$ of nutritional protein. Although potentially beneficial, the percentage increase in protein consumption for calves in the lactoferrin group is small. Considering a 56-d preweaning period, where calves receive $120 \mathrm{~g}$ of protein per day ( $8 \mathrm{~L}$ of whole milk at $\sim 3 \%$ protein), calves in the lactoferrin group would have received only $0.067 \%$ more total dietary protein relative to control group (13,440 vs. 13449 total grams of protein), suggesting that the effect of lactoferrin extends beyond nutritional value alone. The size of the treatment effect in the current study suggests that lactoferrin is economically feasible. Based on this trial, the number needed to treat $(\mathrm{NNT}=1 /$ absolute risk reduction) to prevent at least 1 death or cull is 14.3 for all calves and 6.7 for calves with a fecal score of 4 at onset.

Mitigation of the negative sequelae of diarrheal disease by lactoferrin treatment may plausibly reduce the risk diseases later in the preweaning period. Results suggest that short-term treatment with lactoferrin has a significant and long-term effect on the risk of death and culling. In our study, the risk of death between 60 and $120 \mathrm{~d}$ following enrollment was significantly lower in the lactoferrin group relative to the control group. For calves in the control group, 9.1\% (18/198) of calves died compared with only $1.6 \%(3 / 185)$ of calves in the lactoferrin group $(P<0.05)$. For instance, calves that survive diarrhea weigh less after weaning, have a higher risk for pneumonia, and are 2.5 times more likely to be sold before entering the milking herd (Waltner-Toews et al., 1986; Donovan and Dohoo, 1998). Although we noted a significant effect of lactoferrin on mortality and culling, our study did not demonstrate a significant difference in the shorter-term health outcomes of weight gain and disease duration. The time frame for measurement of weight $(10 \mathrm{~d})$ may have been too short to capture longer-term treatment effects on growth. Survivorship bias may have distorted the true treatment effect on weight gain, as $6 \%(13 / 225)$ of control calves and $3 \%(6 / 199)$ of lactoferrin calves died before the weight measurement at $10 \mathrm{~d}$ following enrollment. Likewise, the time frame for health assessments $(10 \mathrm{~d}$ following enrollment) may have been insufficient for longer term treatment effects that were captured with mortality and culling rates.

The results of our study should not be interpreted to suggest that lactoferrin is a true replacement antimicrobial therapy in cases of calf diarrhea. This study was designed to enroll calves early in the course of disease, and calves with severe dehydration (dehydration score $=2$ ) or significant concurrent diseases were purposefully excluded. Therefore, the results of our study are applicable toward recently diagnosed calves with the first episode of diarrhea with mild or moderate sys- 
temic signs, rather than calves with more severe or advanced disease where conventional parenteral antimicrobial therapy may be necessary to treat septicemia (Fecteau et al., 1997; Lofstedt et al., 1999; Constable, 2004). Additional studies with suitable sample sizes are required to determine if blanket supplementation of all calves would effectively prevent disease or negative health outcomes. Garlic extract numerically lowered the relative risk of death or culling, but the results were not statistically significant. The proportion of calves in the garlic extract and control groups that died or were culled before 60 and $120 \mathrm{~d}$ was 6.8 and 9.8 , respectively (Table 1). Assuming this treatment effect holds across a larger study, over 4,000 calves would need to be enrolled for sufficient statistical power.

\section{CONCLUSIONS}

Lactoferrin significantly reduced mortality and culling when administered to preweaned calves with the first diagnosis of diarrhea, but had no significant effect on disease duration or weight gain. Garlic extract, frequently used by organic producers, did not have a significant effect on any of the health outcomes of the study. Additional studies are necessary to corroborate the observed reduction in mortality and culling in calves that received lactoferrin, and future studies should consider using a larger number of calves across multiple farms, and focus on calves with a fecal score of 4 (watery diarrhea that sifts through the bedding). Additional studies may also be useful to identify effect modifiers, including the initial microbiological cause that might focus the administration of lactoferrin and provide additional insights into the mechanism of action. If the results are confirmed, lactoferrin may become an important tool to improve treatment outcomes and reduce the necessity of antimicrobial use.

\section{ACKNOWLEDGMENTS}

This material is based upon work that is supported by the National Institute of Food and Agriculture, USDA, under award number 2013-38640-20901 through the North Central Region SARE program under subaward number LNC13-351. Any opinions, findings, conclusions, or recommendations expressed in this publication are those of the author(s) and do not necessarily reflect the view of the USDA.

\section{REFERENCES}

Baveye, S., E. Elass, J. Mazurier, and D. Legrand. 2000. Lactoferrin inhibits the binding of lipopolysaccharides to L-selectin and subse- quent production of reactive oxygen species by neutrophils. FEBS Lett. 469:5-8.

Berge, C., D. Moore, T. E. Besser, and W. M. Sischo. 2009. Targeting therapy to minimize antimicrobial use in preweaned calves: Effects on health, growth, and treatment costs. J. Dairy Sci. 92:4707-4714.

Berlutti, F., F. Pantanella, T. Natalizi, A. Frioni, R. Paesano, A. Polimeni, and P. Valenti. 2011. Antiviral properties of lactoferrin-A natural immunity molecule. Molecules 16:6992-7018.

Constable, P. D. 2004. Antimicrobial use in the treatment of calf diarrhea. J. Vet. Intern. Med. 18:8-17.

Cowles, K. E., R. White, N. L. Whitehouse, and P. S. Erickson. 2006 Growth characteristics of calves fed an intensified milk replacer regimen with additional lactoferrin. J. Dairy Sci. 89:4835-4845.

Donovan, G. A., and I. Dohoo. 1998. Calf and disease factors affecting growth in female Holstein calves in Florida, USA. Prev. Vet. Med. 33:1-10.

Dutil, L. 2010. Ceftiofur resistance in Salmonella enterica serovar Heidelberg from chicken meat and humans, Canada. Emerg. Infect. Dis. 16:48-54.

Elass-Rochard, E., D. Legrand, V. Salmon, A. Roseanu, M. Trif, P. S. Tobias, J. Mazurier, and G. Spik. 1998. Lactoferrin inhibits the endotoxin interaction with CD14 by competition with the lipopolysaccharide-binding protein. Infect. Immun. 66:486-491.

Ellison, R. T., and T. J. Giehl. 1991. Killing of gram-negative bacteria by lactoferrin and lysozyme. J. Clin. Invest. 88:1080-1091.

English, E. A., B. A. Hopkins, J. S. Stroud, S. Davidson, G. Smith, C. Brownie, and L. W. Whitlow. 2007. Lactoferrin supplementation to Holstein calves during the preweaning and postweaning phases. J. Dairy Sci. 90:5276-5281.

Fecteau, G., J. Paré, D. C. Van Metre, B. P. Smith, C. A. Holmberg, W. Guterbock, and S. Jang. 1997. Use of a clinical sepsis score for predicting bacteremia in neonatal dairy calves on a calf rearing farm. Can. Vet. J. 38:101-104.

Flores-Villaseñor, H., A. Canizalez-Román, J. Velazquez-Roman, K. Nazmi, J. G. M. Bolscher, and N. Leon-Sicairos. 2012. Protective effects of lactoferrin chimera and bovine lactoferrin in a mouse model of enterohaemorrhagic Escherichia coli O157:H7 infection. Biochem. Cell Biol. 90:405-411.

Ghosh, S., R. K. Mehla, S. K. Sirohi, and B. Roy. 2010. The effect of dietary garlic supplementation on body weight gain, feed intake, feed conversion efficiency, faecal score, faecal coliform count and feeding cost in crossbred dairy calves. Trop. Anim. Health Prod. 42:961-968

Ghosh, S., R. K. Mehla, S. K. Sirohi, and S. K. Tomar. 2011. Performance of crossbred calves with dietary supplementation of garlic extract. J. Anim. Physiol. Anim. Nutr. (Berl.) 95:449-455.

Habing, G., C. Djordjevic, G. M. Schuenemann, and J. Lakritz. 2016. Understanding antimicrobial stewardship: Treatment thresholds and alternatives among conventional and organic herds. Prev. Vet. Med. 130:77-85.

Isaacson, R. E., H. W. Moon, and R. A. Schneider. 1978. Distribution and virulence of Escherichia coli in the small intestines of calves with and without diarrhea. Am. J. Vet. Res. 39:1750-1755.

Joslin, R. S., P. S. Erickson, H. M. Santoro, N. L. Whitehouse, C. G. Schwab, and J. J. Rejman. 2002. Lactoferrin supplementation to dairy calves. J. Dairy Sci. 85:1237-1242.

Lofstedt, J., I. R. Dohoo, and G. Duizer. 1999. Model to predict septicemia in diarrheic calves. J. Vet. Intern. Med. 13:81-88.

Lombard, J. E., C. P. Fossler, A. E. Adams, and C. A. Kopral. 2015. Use of antibiotics on U.S. dairy operations. Pages 298-299 in Proceedings of the 2015 Conference of The American Association of Bovine Practitioners.

McGuirk, S. M. 2008. Disease management of dairy calves and heifers. Vet. Clin. North Am. Food Anim. Pract. 24:139-153.

Moon, H. W., A. W. McClurkin, R. E. Isaacson, J. Pohlenz, S. M. Skartvedt, K. G. Gillette, and A. L. Baetz. 1978. Pathogenic relationships of rotavirus, Escherichia coli, and other agents in mixed infections in calves. J. Am. Vet. Med. Assoc. 173:577-583.

Prenner, M. L., C. Prgomet, H. Sauerwein, M. W. Pfaffl, J. Broz, and F. J. Schwarz. 2007. Effects of lactoferrin feeding on growth, feed intake and health of calves. Arch. Anim. Nutr. 61:20-30. 
Robblee, E. D., P. S. Erickson, N. L. Whitehouse, A. M. McLaughlin, C. G. Schwab, J. J. Rejman, and R. E. Rompala. 2003. Supplemental lactoferrin improves health and growth of Holstein calves during the preweaning phase. J. Dairy Sci. 86:1458-1464.

Saini, V., J. T. McClure, D. T. Scholl, T. J. DeVries, and H. W. Barkema. 2012. Herd-level association between antimicrobial use and antimicrobial resistance in bovine mastitis Staphylococcus aureus isolates on Canadian dairy farms. J. Dairy Sci. 95:1921-1929.

Still, J., P. Delahaut, and P. Coppe. 1990. Treatment of induced enterotoxigenic colibacillosis (scours) in calves by the lactoperoxidase system and lactoferrin. Ann. Rech. Vet. 21:143-152.

Turin, C. G., A. Zea-Vera, A. Pezo, K. Cruz, J. Zegarra, S. Bellomo, L. Cam, R. Llanos, A. Castañeda, L. Tucto, T. J. Ochoa, and NEOLACTO Research Group. 2014. Lactoferrin for prevention of neonatal sepsis. Biometals 27:1007-1016.

USDA. 2010. Heifer Calf Health and Management Practices on US Dairy Operations, 2007. USDA Animal and Plant Health Inspection Service, Veterinary Service, Center for Epidemiology and Animal Health, Fort Collins, CO. van Leeuwen, P., S. J. Oosting, J. M. V. M. Mouwen, and M. W. A. Verstegen. 2000. Effects of a lactoperoxidase system and lactoferrin, added to a milk replacer diet, on severity of diarrhoea, intestinal morphology and microbiology of digesta and faeces in young calves. J. Anim. Physiol. Anim. Nutr. (Berl.) 83:15-23.

Waltner-Toews, D., S. Martin, and A. Meek. 1986. Dairy calf management, morbidity and mortality in ontario hostein herds. III. Association of management with morbidity. Prev. Vet. Med. 4:137-158.

Weinberg, E. D. 2001. Human lactoferrin: A novel therapeutic with broad spectrum potential. J. Pharm. Pharmacol. 53:1303-1310.

Zavaleta, N., D. Figueroa, J. Rivera, J. Sánchez, S. Alfaro, and B. Lönnerdal. 2007. Efficacy of rice-based oral rehydration solution containing recombinant human lactoferrin and lysozyme in Peruvian children with acute diarrhea. J. Pediatr. Gastroenterol. Nutr. $44: 258-264$.

Zou, G. 2004. A modified Poisson regression approach to prospective studies with binary data. Am. J. Epidemiol. 159:702-706. 